\title{
Transcription activity of transposon sequence limits Sleeping Beauty transposition
}

\author{
Orsolya Kolacsek ${ }^{a}$ and Tamás I. Orbán ${ }^{\text {a,* }}$ \\ ${ }^{a}$ Institute of Enzymology, Research Centre for Natural Sciences, Hungarian Academy of \\ Sciences, Budapest, Hungary
}

*Correspondence to:

Tamás I. Orbán

Institute of Enzymology, Research Centre for Natural Sciences,

Hungarian Academy of Sciences

1117 Budapest, Magyar Tudósok krt. 2.

Hungary

Phone: +3613826638

Fax: +3613826295

Email: orban.tamas@ttk.mta.hu

Keywords: Sleeping Beauty; piggyBac; promoter; copy number

Abbreviations: SB (Sleeping Beauty); PB (piggyBac) 


\begin{abstract}
Sleeping Beauty (SB) transposon based technology has been extensively applied in basic research and biotechnology for routine cell culture gene delivery and vertebrate transgenesis, and it is also investigated in various gene therapy applications. Cell tolerance for the transgene is a key factor during transgenesis and is modulated not only through the type but by the dose of expression. Our experimental results exemplify that transgenes regulated with high activity promoters can reduce the overall success of gene delivery. Observations connected to transposon donors regulated by different promoters have also revealed inverse correlation between transcription activity and the hyperactive variant SB100X excision efficiency. This competition between transcription and transposition was independent of the transgene coding sequence and did not alter the transgenic efficiency in general. However, promoters applied in the transgene cassette can produce different average copy numbers depending on the transcriptional activity of the transposon. Unlike the piggyBac (PB) transposon system, this phenomenon allows a fine balance of expression using the high copy potential SB system that adjusts the copy number of lower activity promoter driven transgenes to a higher expression level. All this contributes to a well-tolerated and satisfactory transgenesis, and would be important to consider in gene therapy applications.
\end{abstract}




\section{Introduction}

Mobility of transposons can be utilized for gene transfer in both experimental and therapeutic applications. Among the DNA transposon systems, the Sleeping Beauty (SB) transposon is a reconstructed element from fish genomes and it is one of the bestcharacterized transposons in terms of activity in vertebrate cells (Kawakami et al., 2017; Narayanavari et al., 2017). The most active SB100X variant supports gene integration at a level approaching the efficiency of integrating viral vector systems (Mates et al., 2009). SB has a close-to-random integration profile, which contributes to its enhanced safety in therapeutic applications (Gogol-Doring et al., 2016; Kebriaei et al., 2017). In addition, its relative independence on species or cell type specific cellular factors allows its application in a wide range of cell types, including various stem cells.

Efficient genomic integration of SB enables stable expression of large variety of transgenes as exemplified by several preclinical animal models and cell culture studies (Izsvak et al., 2010). It is generally accepted that the size of the transgene lowers transposition efficiency, although BAC clones over the size of $150 \mathrm{~kb}$ can be stably integrated by the SB system into vertebrate genomes (Rostovskaya et al., 2012; Wang et al., 2014). However, cellular tolerance to transgenesis can vary depending on the type and expression level of the transgene which might also modulate the overall efficiency or outcome of transgenesis. This is to say that the nature of the promoter and the transgene are not expected to influence transposition rates, rather their effects are assumed to be manifested by the interference with the cellular expression machinery and by the genomic defense activity of the particular cell type. Different transgenes can be tolerated differently in a cell type specific manner, so the expression dose is recommended to be carefully adjusted to avoid this selection effect. This is often achieved by the appropriate choice of the promoter, and some of our previous 
experiments supported this idea: certain viral promoters (e.g. CMV, cytomegalovirus promoter) are strongly silenced in human embryonic stem cells as compared to other constitutive promoters (Orban et al., 2009), suggesting that lower activity promoters, especially those ones derived from endogenous sequences, could lead to a more stable long term transgene expression.

Contrary to that, when optimizing the parameters of the SB and the piggyBac (PB) transposon systems, some experiments indicated that transcription activity of different promoters may additionally influence transposition efficiencies (Kolacsek et al., 2014). Follow up studies of long term cell culture experiments revealed that, particularly for the hyperactive SB100X transposase, transposon copy numbers are influenced by the choice of promoters, as if there was a balancing effect on the overall transgene expression. Since there was some indications that a strong promoter activity could interfere with SB transposition efficiency, this important aspect of transposon-based gene delivery awaited further careful examinations. Therefore, in this study we have experimentally tested how transgene expression provided by different activity promoters in the delivered transposon cassette could affect transposition efficiency. We have also addressed the question of which step of the transposition reaction is affected by the level of transcription, and what would the overall outcome of this interference have on the resulting transgenic cell number.

\section{Materials and methods}

\subsection{Plasmid constructs}


SB100X transposase variant was expressed from a standard expression plasmid regulated by a CMV promoter (pCMV-SB100X) (Mates et al., 2009). SB transposon donor plasmids were created from the pT2 variant of the SB inverted repeat-direct repeats (IRDRs) (Geurts et al., 2003). Puromycin antibiotic resistance gene was cloned into the initial transposon plasmid with PCR technique; CAG, CMV, PGK, and SV40 promoters were cloned with standard restriction cassette replacements. For GFP expression plasmids, the puromycin coding sequences in these transposon donor plasmids were changed to GFP using restriction enzymes. For transfection control, the pCMV-EGFP (Clontech) expression plasmid was used.

\subsection{Cell maintenance and transfection method}

Human embryonic kidney cells (HEK-293) were cultured in Dulbecco's modified Eagle's medium (DMEM) supplemented with 10\% fetal calf serum, 1\% 1-glutamine, and 1\% penicillin-streptomycin. $2 \times 10^{5}$ HEK-293 cells were seeded onto 12-well plates, and transfected with FuGENE 6 reagent (Promega) in duplicate according to the manufacturer's instructions. For colony assays, 10:1 or 1:10 donor - helper dose combinations were applied, e.g. $400 \mathrm{ng}$ puromycin containing transposon donor plasmids were co-transfected with $40 \mathrm{ng}$ pCMV-SB100X helper vector or vice versa. All transfections were supplemented with $400 \mathrm{ng}$ pCMV-EGFP transfection control. Two days posttransfection cells were harvested and one tenth of the transfections were analyzed with Attune Acoustic Focusing Cytometer (Thermo Fisher Scientific) for GFP expressing cells. Transfection efficiencies were comparable within experiments, ranging from $72 \%$ to $84 \%$.

\subsection{Transposition assay}


For colony forming assay, 48 hours posttransfection $1 \%$ of the transfected cells were seeded onto cell culture Petri dishes and selected for 3 weeks with $1 \mu \mathrm{g} / \mathrm{ml}$ puromycin. Surviving cells were fixed with methanol and stained with $0.05 \%$ crystal violet in $25 \%$ methanol. Colonies were quantified with a universal hood gel imager model 75S, using Quantity One 4.4.0 software (Bio-Rad, Hercules, CA). Transgenic rates were calculated from the colony numbers, normalizing with the number of seeded cells and also for transfection efficiencies; transgenic rates are therefore defined as a percentage of transfected cells.

\subsection{Transposon copy number measurements}

For average copy number quantification, cells were harvested two days posttransfection and one tenths of the cells in parallels were selected in puromycin for 3 weeks. Cells from parallels were pooled and genomic DNAs were isolated by the standard

phenol-chloroform extraction method. Average copy numbers were quantified with a $\operatorname{TaqMan}^{\circledR}$ assay specific for the IRDR-L (left transposon sequence), normalizing to the level of the RPPH1 endogenous control gene as described previously (Kolacsek et al., 2011). Briefly, all samples were run in triplicate singleplex reactions, using $100 \mathrm{ng}$ of gDNA template, in a final volume of $20 \mu \mathrm{l}$. Real-time PCR measurements were performed on a StepOnePlus $^{\mathrm{TM}}$ platform, and data were analyzed by the StepOne software version 2.1, according to the manufacturer's instructions (Thermo Fisher Scientific).

\subsection{Expressivities of the promoters}


HEK-293 cells were transfected as described above, except that 800ng GFP transposon donor plasmids were solely applied. Two days posttransfection cells were harvested and parallels were pooled. GFP expressing cells were separated with FACSAria flow cytometer according to the manufacturer's instructions (Becton, Dickinson and Company) and seeded onto a 12 -well-plate. Next day they were lysed with $500 \mu 1$ peqGOLD TriFast (VWR Peqlab) and total RNAs were extracted by standard protocol of the manufacturer. RNAs were treated with DNase I to remove plasmid DNA contamination. Reverse transcriptions were carried out with High-Capacity cDNA Reverse Transcription kit (Thermo Fischer Scientific) according to the manufacturer's instructions, including negative controls without adding reverse transcriptase. For qPCR reactions, $5 \mu$ of tenfold diluted cDNAs were applied in $20 \mu \mathrm{l}$ reaction volume in triplicates. Reactions were carried out with $\operatorname{TaqMan}^{\circledR}$ Gene Expression Master Mix (Thermo Fisher Scientific), and were run on StepOnePlus real-time PCR system platform using standard protocol; results were analyzed by StepOne software version 2.1. TaqMan ${ }^{\circledR}$ primers and probe for the GFP were: forward primer, 5'-GAGCGCACCATCTTCTTCAAG; reverse primer, 5'TGTCGCCCTCGAACTTCAC; TaqMan ${ }^{\circledR}$ labeled probe, 5'-ACGACGGCAACTACA. Final concentrations of primers and probes were $900 \mathrm{nM}$ and $250 \mathrm{nM}$, respectively. Ribosomal protein P0 (RPLP0, TaqMan ${ }^{\circledR}$ assay Hs9999902_m1) was used as endogenous control and the $\Delta \Delta \mathrm{Ct}$ method was applied for relative quantification.

\subsection{Excision activities}

To quantitate the excision efficiency of the hyperactive SB100X transposase on different transposon donors, HEK-293 cells were co-transfected with $400 \mathrm{ng}$ of transposon donor and $400 \mathrm{ng}$ of SB100X expressing helper plasmids. Two days posttransfection cells were 
harvested and parallels were pooled. Isolation of plasmid DNA and real-time qPCRs were carried out essentially as described previously (Kolacsek et al., 2014). Briefly, the Qiagen plasmid miniprep kit was used for DNA isolation with a modified protocol: the cell lysis/protein removal step solution was supplemented with $300 \mu \mathrm{l}$ of $1.2 \%$ sodium dodecyl sulfate solution and with $50 \mu \mathrm{g}$ of proteinase K. $10 \mathrm{ng}$ of the purified DNA samples were used as templates in a two-round nested PCR where primers specific for the transposon donor plasmid backbone were used, amplifying sequences from post-excision plasmids that underwent DNA repair. Plasmids without excision do not yield PCR amplicons due to the large size of the transposon cassettes. The second round of the nested PCR is carried out on a StepOnePlus $^{\mathrm{TM}}$ real-time PCR platform, using SYBR Green chemistry (Thermo Fisher Scientific). For normalization, PCR primers specific for the ampicillin sequence were used which is exclusively present on the transposon donor plasmid; excision efficiencies were then calculated by the $\Delta \Delta \mathrm{Ct}$ method.

\section{Results and Discussion}

\subsection{Choice of promoters influence SB transgene copy numbers}

Our previous works with the most frequently and routinely applied two-component SB transfection system have showed that dose combinations of transposon donor and transposase expressing helper plasmids define different average transgene copy numbers after cotransfection and selection of transgenic cells (Kolacsek et al., 2014). Ten to one (10:1) donor - helper ratio resulted in the highest average copy number, while one to ten $(1: 10)$ dose combination supported transgenic cell population with low average copy number and 
comparably high transgenic efficiency when hyperactive SB100X was applied. Therefore these two dose combinations providing high and low copy numbers are well suitable to monitor the cell tolerance toward different promoters in the transgenesis. Four frequently used promoters were cloned into SB transgene cassettes expressing puromycin antibiotic resistance gene. These were the CAG composite promoter with CMV (cytomegalovirus) enhancer and two different chicken- and rabbit endogenous promoter elements, the CMV promoter, PGK(phosphoglycerate kinase), and SV40 (simian virus 40) promoter. Evaluating the transgene delivery experiments in HEK-293 cells with colony assays, transgenic cell numbers were not considerably different between promoters when low transposon donor dose was applied (Figure 1A, 1:10 donor-helper ratio). It suggests that low transgene load is well tolerated independent of the type of the promoter regulating the transgene expression. More fluctuations were seen among promoters at high transposon donor dose (Figure 1A, 10:1 donor-helper ratio), and CMV-puromycin transgene cassette resulted the lowest number of transgenic colonies suggesting lower tolerance of cells for CMV driven transgene expression. Moreover, when we compare the two dose combinations (10:1 vs. 1:10), in the case of CMV two fold difference is observed in transgenic efficiency, while the tolerance of the other types of promoters did not seem to depend on transposon load (Figure 1A).

We also examined the average copy numbers of these selected transgenic cell populations which revealed unexpected differences between the examined promoters (Figure 1B). Copy numbers produced by the high activity CAG and CMV promoter driven transposons were significantly lower at both dose combinations than in the cases of PGK and SV40 promoters. Next we have verified the expression activity of these promoters by measuring transcription with qRT-PCR. Using GFP as the transgene with different promoters, cells transfected with the SB constructs were sorted for GFP expressing cells by FACS two days following transfection, and then GFP mRNA levels were measured in those cells. CAG 
and CMV promoters showed much higher transcription activities compared to PGK and SV40 (Figure 2).

\subsection{Promoter activity influences $S B$ excision rate}

At this point one could expect that differences in copy numbers of selected cells merely represent lower tolerance of higher transgene expression levels. This might be true in the case of CAG and CMV promoter. However, the much lower activity PGK and SV40 promoters also showed at least two fold difference in copy numbers at both dose combinations, and expression levels produced by them differentiated to a similar extent (Figure 1B and Figure 2). This raises the possibility that transposase function can be directly influenced by the expression activity of a particular promoter in the transposon. Cell tolerance level for an exogenous protein expression is difficult to determine, but definitely detectable over a longer period of time. Immediate transposase activity in co-transfected cells is certainly not dependent on cell tolerance that develops later during delivery and selection of transgenic cells. Transposase activity is often characterized by measuring the transposon excision product left behind after excision and reparation of double stranded breaks on the donor plasmid (Mates et al., 2009; Yusa et al., 2011; Claeys Bouuaert et al., 2013; Kolacsek et al., 2014). Quantification of excised and re-circularized donor plasmids by real-time PCR proved the transcription dependence of transposase activity (Figure 3). Transfecting the two component SB100X transposon system into HEK-293 cells, the amount of excision products inversely correlated with the transcription activity of promoters applied in the transposon cassette (compare Figure 2 and 3). Transposase activity correlates more obviously with the resulting copy number (Figure 3 and Figure 1B). This phenomenon was independent of the 
type of the transgene, as both puromycin and GFP expressing transposon showed similar excision tendency between the examined promoters (Figure 3).

These promoters are intended to express the transgene from a genomic locus after delivery; however, they also constantly regulate transcription frequency ectopically from the plasmid before chromosomal integration. We can draw the conclusion that this episomal expression could limit transposition efficiency. This idea is supported by earlier observations when donor plasmids methylated in vitro allows more efficient delivery in mice transgenesis, and the transgene will be released from repression thereafter during epigenetic reprogramming of embryonic stem cells (Carlson et al., 2011).

Our experimental data led to the assumption that continuous presence of the transcriptional machinery owing to higher activity promoters may develop steric hindrance for transposition of an actively transcribed DNA substrate. We expected this competition between transcription and transposition to be a ubiquitous function of DNA transposons. However, in the case of similarly widely used piggyBac (PB) transposon system, representing a different superfamily of DNA transposases, we could not reproduce this phenomenon (data not shown), suggesting that $\mathrm{PB}$ transposition may also be sensitive to other factors binding to different promoters. Nevertheless we may conclude that both transposon systems are similarly efficient in establishing transgenic cells independently of promoter sequence, but SB tends to make different copy numbers depending on the transcription activity of the transgene and on the transposon donor load in delivery (Figure 1B). In connection with the latter aspect of SB100X, promoters with extremely high activity (like the viral-derived CMV promoter) are not recommended to be applied in high transposon donor dose because the expected high copy numbers can reduce transgenic cell number owing to bad tolerance for high level of transgene expression (Figure 1A). Furthermore, a remarkable selective disadvantage appears 
for CMV driven highly expressing transgenic cells when the population is not under selective pressure (unpublished data).

Competition between transcription and SB transposition allows balancing the overall expression level of transgene, as lower activity promoters can in fact produce higher transgene expression owing to higher copy number; nevertheless, this also depends on the dose of transposon donor and is also balanced by the tolerability of the protein. While PB generally produce much lower copy numbers (Grabundzija et al., 2010; Kolacsek et al., 2014), the high copy "potential" of SB gives the opportunity for fine adjustment of copy number in delivery of transgene, and in addition, it makes SB quite suitable for multiple gene delivery (Kacherovsky et al., 2015). Based on our studies, for increased efficiency of multiple transgene delivery applications, using lower activity promoters is worth to consider.

\subsection{Conclusions}

Using several widely used eukaryotic promoters in SB transposon cassettes, we concluded that the transcription activity of the promoters applied in the transposon cassette interfere with SB100X transposition, and this interference is manifested at the excision step of transposition. The competition between transcription and transposition elevates the transgene copy numbers of lower activity promoters during transgenesis and as a result, the overall transgene expression level will be higher from weaker promoters. This "fine-tuning" of the gene expression produces similar expression levels from lower copy number of strong promoter-driven transgenes that would otherwise be less tolerated for the cells. Based on these new observations, we could provide an "updated user guide" for SB transposon delivery applications, in order to achieve the most optimal long term transgene expression levels. As a 
first issue, transfection efficiency should be taken into considerations: at low efficiency rate (e.g. for hard-to-transfect cell lines), high transposon donor dose will result in sufficient number of transgenic cells, and can produce low average transgene copies per cell. On the other hand, in cases of higher transfection efficiency, the application of strong promoters (like CMV), especially at high donor doses, can be unfavorable due to the potential of the transgene silencing, as well as to the bad tolerance of cells towards highly expressing copies, reducing transgenic efficiency. In such cases, promoters with moderate or low transcription activity (typically from endogenous promoters) can be beneficial, as they are mobilized more effectively due to the less interference with transposition, and the level of expression can be optimal with higher number of integrated transgene copies. When transgene expression level needs more adjustments, transposon donor dosage can be used to further manipulate the resulting copy number. At high transfection rates, increasing the dose of transposase might help in increasing transposition efficiency, however, although not observed in our experiments, this could have an adverse effect due to the overproduction inhibition phenomenon (Bire et al., 2013).

\section{Declarations of interest}

None.

\section{Acknowledgments}

The authors are grateful to Kornélia Némethy for excellent technical assistance. 


\section{Funding}

Tamás I. Orbán is a recipient of the János Bolyai Scholarship of the Hungarian Academy of Sciences. This work was supported by the Hungarian Scientific Research Fund (NKFIHOTKA, grant number K112112), and "Szinergia VIII. - MedInProt" program.

\section{Authors' contributions}

OK: designed the study, performed the experiments, analyzed the data, wrote the manuscript TIO: designed the study, analyzed the data, wrote and finalized the manuscript

\section{References}

Bire, S., Casteret, S., Arnaoty, A., Piegu, B., Lecomte, T. and Bigot, Y., 2013. Transposase concentration controls transposition activity: myth or reality? Gene 530, 165-71.

Carlson, D.F., Geurts, A.M., Garbe, J.R., Park, C.W., Rangel-Filho, A., O'Grady, S.M., Jacob, H.J., Steer, C.J., Largaespada, D.A. and Fahrenkrug, S.C., 2011. Efficient mammalian germline transgenesis by cis-enhanced Sleeping Beauty transposition. Transgenic Res 20, 29-45.

Claeys Bouuaert, C., Lipkow, K., Andrews, S.S., Liu, D. and Chalmers, R., 2013. The autoregulation of a eukaryotic DNA transposon. eLife 2, e00668. 
Geurts, A.M., Yang, Y., Clark, K.J., Liu, G., Cui, Z., Dupuy, A.J., Bell, J.B., Largaespada, D.A. and Hackett, P.B., 2003. Gene transfer into genomes of human cells by the sleeping beauty transposon system. Mol Ther 8, 108-17.

Gogol-Doring, A., Ammar, I., Gupta, S., Bunse, M., Miskey, C., Chen, W., Uckert, W., Schulz, T.F., Izsvak, Z. and Ivics, Z., 2016. Genome-wide Profiling Reveals Remarkable Parallels Between Insertion Site Selection Properties of the MLV Retrovirus and the piggyBac Transposon in Primary Human CD4(+) T Cells. Mol Ther 24, 592-606.

Grabundzija, I., Irgang, M., Mates, L., Belay, E., Matrai, J., Gogol-Doring, A., Kawakami, K., Chen, W., Ruiz, P., Chuah, M.K., VandenDriessche, T., Izsvak, Z. and Ivics, Z., 2010. Comparative analysis of transposable element vector systems in human cells. Mol Ther 18, 1200-9.

Kacherovsky, N., Liu, G.W., Jensen, M.C. and Pun, S.H., 2015. Multiplexed gene transfer to a human T-cell line by combining Sleeping Beauty transposon system with methotrexate selection. Biotechnol Bioeng 112, 1429-36.

Kawakami, K., Largaespada, D.A. and Ivics, Z., 2017. Transposons As Tools for Functional Genomics in Vertebrate Models. Trends Genet 33, 784-801.

Kebriaei, P., Izsvak, Z., Narayanavari, S.A., Singh, H. and Ivics, Z., 2017. Gene Therapy with the Sleeping Beauty Transposon System. Trends Genet 33, 852-870.

Kolacsek, O., Erdei, Z., Apati, A., Sandor, S., Izsvak, Z., Ivics, Z., Sarkadi, B. and Orban, T.I., 2014. Excision efficiency is not strongly coupled to transgenic rate: cell typedependent transposition efficiency of sleeping beauty and piggyBac DNA transposons. Hum Gene Ther Methods 25, 241-52. 
Kolacsek, O., Krizsik, V., Schamberger, A., Erdei, Z., Apati, A., Varady, G., Mates, L., Izsvak, Z., Ivics, Z., Sarkadi, B. and Orban, T.I., 2011. Reliable transgene-independent method for determining Sleeping Beauty transposon copy numbers. Mobile DNA 2, 5.

Mates, L., Chuah, M.K., Belay, E., Jerchow, B., Manoj, N., Acosta-Sanchez, A., Grzela, D.P., Schmitt, A., Becker, K., Matrai, J., Ma, L., Samara-Kuko, E., Gysemans, C., Pryputniewicz, D., Miskey, C., Fletcher, B., Vandendriessche, T., Ivics, Z. and Izsvak, Z., 2009. Molecular evolution of a novel hyperactive Sleeping Beauty transposase enables robust stable gene transfer in vertebrates. Nat Genet 41, 753-61.

Narayanavari, S.A., Chilkunda, S.S., Ivics, Z. and Izsvak, Z., 2017. Sleeping Beauty transposition: from biology to applications. Crit Rev Biochem Mol Biol 52, 18-44.

Orban, T.I., Apati, A., Nemeth, A., Varga, N., Krizsik, V., Schamberger, A., Szebenyi, K., Erdei, Z., Varady, G., Karaszi, E., Homolya, L., Nemet, K., Gocza, E., Miskey, C., Mates, L., Ivics, Z., Izsvak, Z. and Sarkadi, B., 2009. Applying a "double-feature" promoter to identify cardiomyocytes differentiated from human embryonic stem cells following transposon-based gene delivery. Stem Cells 27, 1077-87.

Rostovskaya, M., Fu, J., Obst, M., Baer, I., Weidlich, S., Wang, H., Smith, A.J., Anastassiadis, K. and Stewart, A.F., 2012. Transposon-mediated BAC transgenesis in human ES cells. Nucleic Acids Res 40, e150.

Wang, Y., Wang, J., Devaraj, A., Singh, M., Jimenez Orgaz, A., Chen, J.X., Selbach, M., Ivics, Z. and Izsvak, Z., 2014. Suicidal Autointegration of Sleeping Beauty and piggyBac Transposons in Eukaryotic Cells. PLoS Genet 10, e1004103.

Yusa, K., Zhou, L., Li, M.A., Bradley, A. and Craig, N.L., 2011. A hyperactive piggyBac transposase for mammalian applications. Proc Natl Acad Sci U S A 108, 1531-6. 


\section{Figure Legends}

Figure 1. Transgenic efficiencies of SB100X in HEK-293 cells depending on promoters of the transgene, as well as on transposon / transposase dose combinations.

Comparison of four frequently used promoters in puromycin resistance gene containing transposon deliveries, using high and low transposon doses. (A) The structure of the used expression cassettes differ only by the indicated promoter sequences. (B-C) Transgenic rates determined by colony assays, and photos of CMV driven transgene colonies (two parallels shown for each dose). Error bars represent standard deviations of parallel transfections. For statistical analysis, two-sided Student's t-tests were applied, **: $\mathrm{p}<0.01$. (D) Average copy number quantification of the same deliveries by qPCR specific for the SB transposon IRDR-L sequence. Error bars represent 95\% confidence intervals of parallel measurements.

Figure 2. Comparison of promoter activities in HEK-293 cells by qRT-PCR.

Four different promoters driven GFP containing SB transposon donor plasmids were transfected and sorted by FACS two days following transfection. Messenger RNA levels were measured by qPCR assay specific for GFP. No RT controls were performed in each case, and no amplifications were detected for either the endogenous control (P0) or GFP. Error bars represent $95 \%$ confidence intervals of parallel measurements. Two-sided Student's t-tests were performed for statistical analysis, $* *: \mathrm{p}<0.01$, ***: $\mathrm{p}<0.001$.

Figure 3. Excision activities of SB100X in HEK-293 cells depending on promoters of the transgene.

Comparison of four different promoters driven transposon donor excision with relative quantification by qPCR. Error bars represent $95 \%$ confidence intervals of parallel 
measurements. For statistical analysis, two-sided Student's t-tests were applied; ${ }^{* *}: \mathrm{p}<0.01$, $* * *: \mathrm{p}<0.001$ 
(A) Structure of the transgenic cassettes

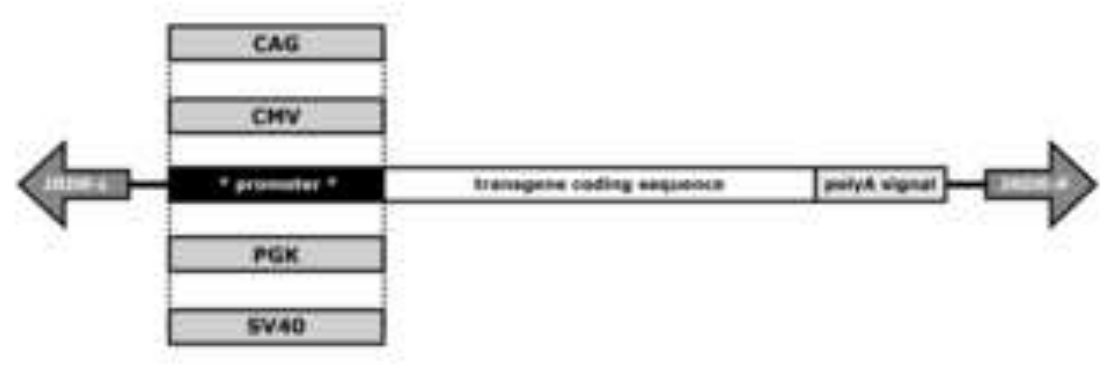

(C) Transgenic rates

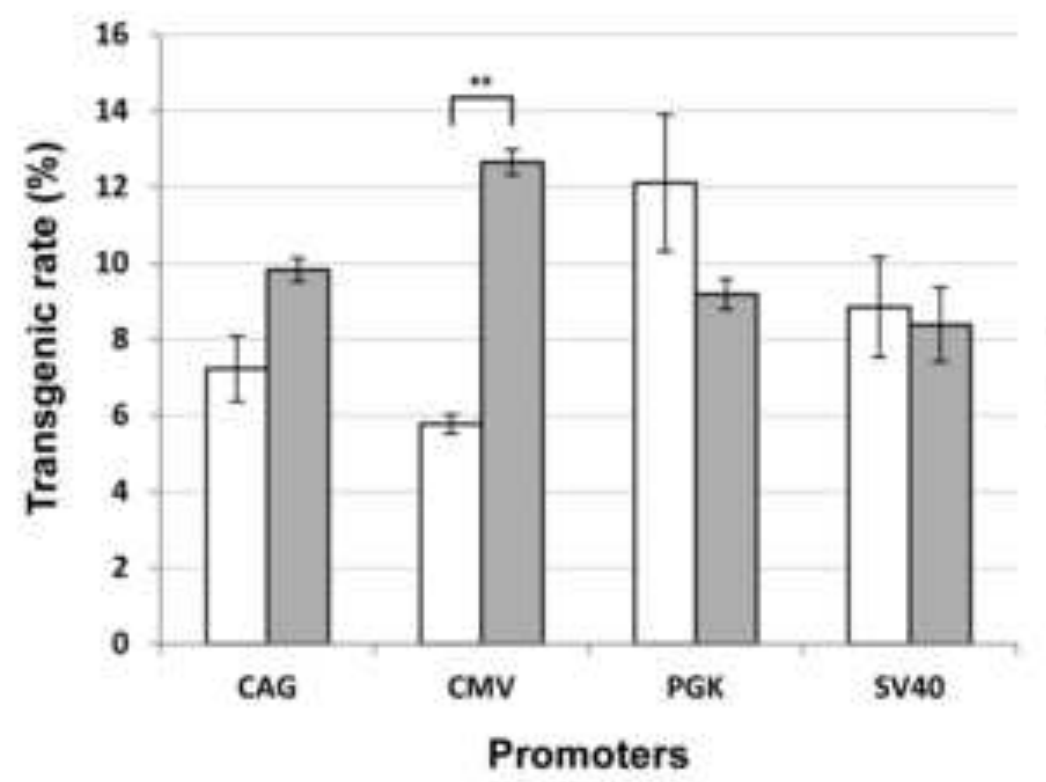

(B) Representative colony assays SB-CMV-puromycin transposon / SB100X transposase rate

$10: 1$

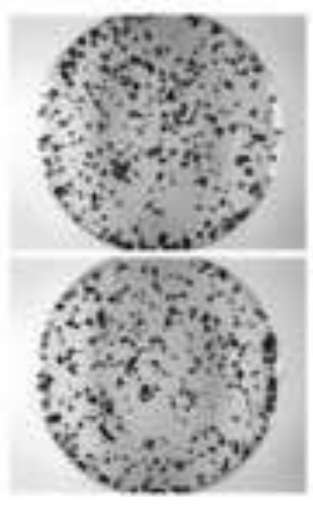

$1: 10$

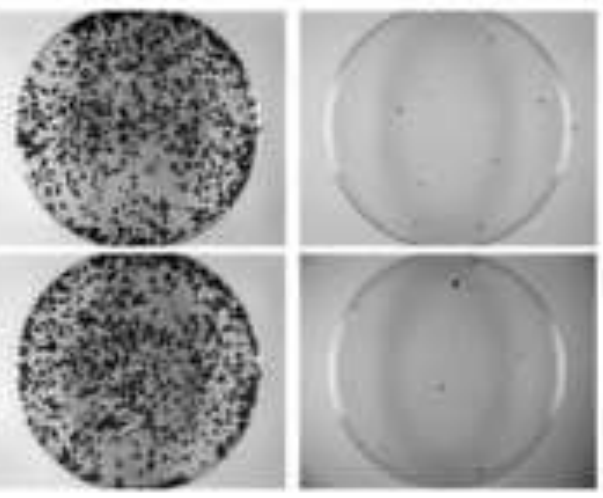

(D) Average copy numbers

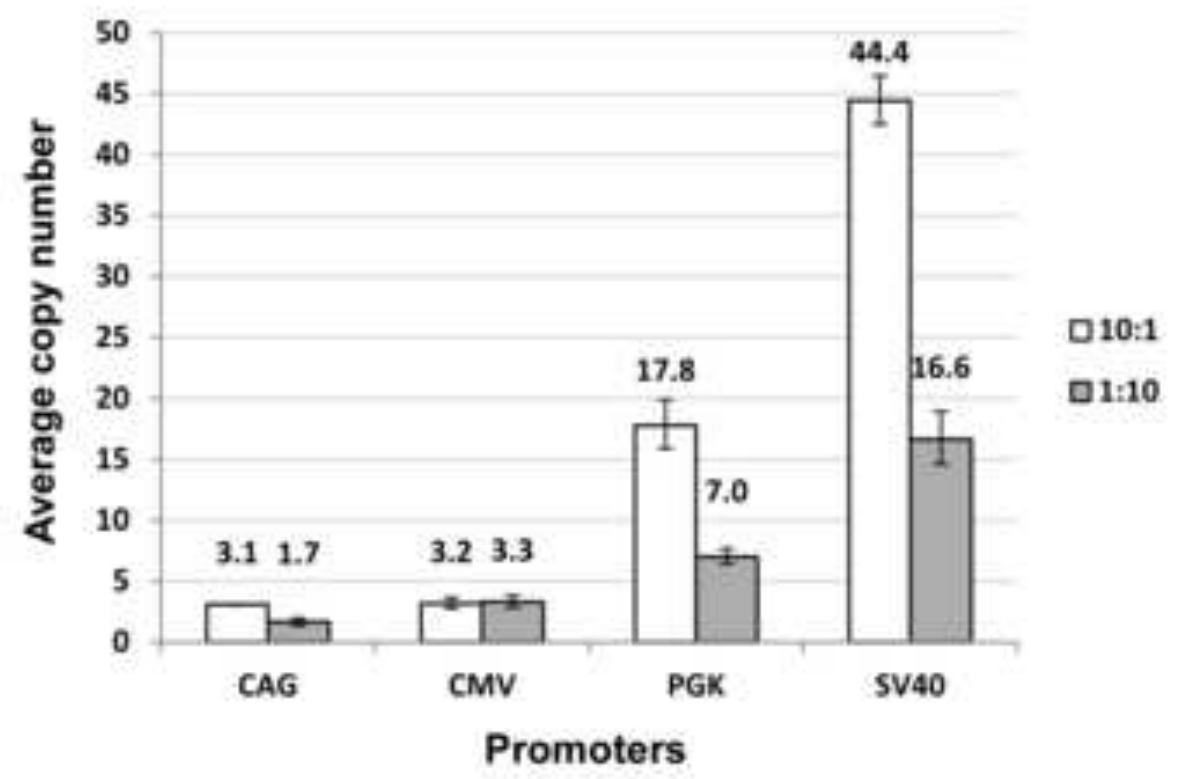

Figure 1 
Click here to download high resolution image

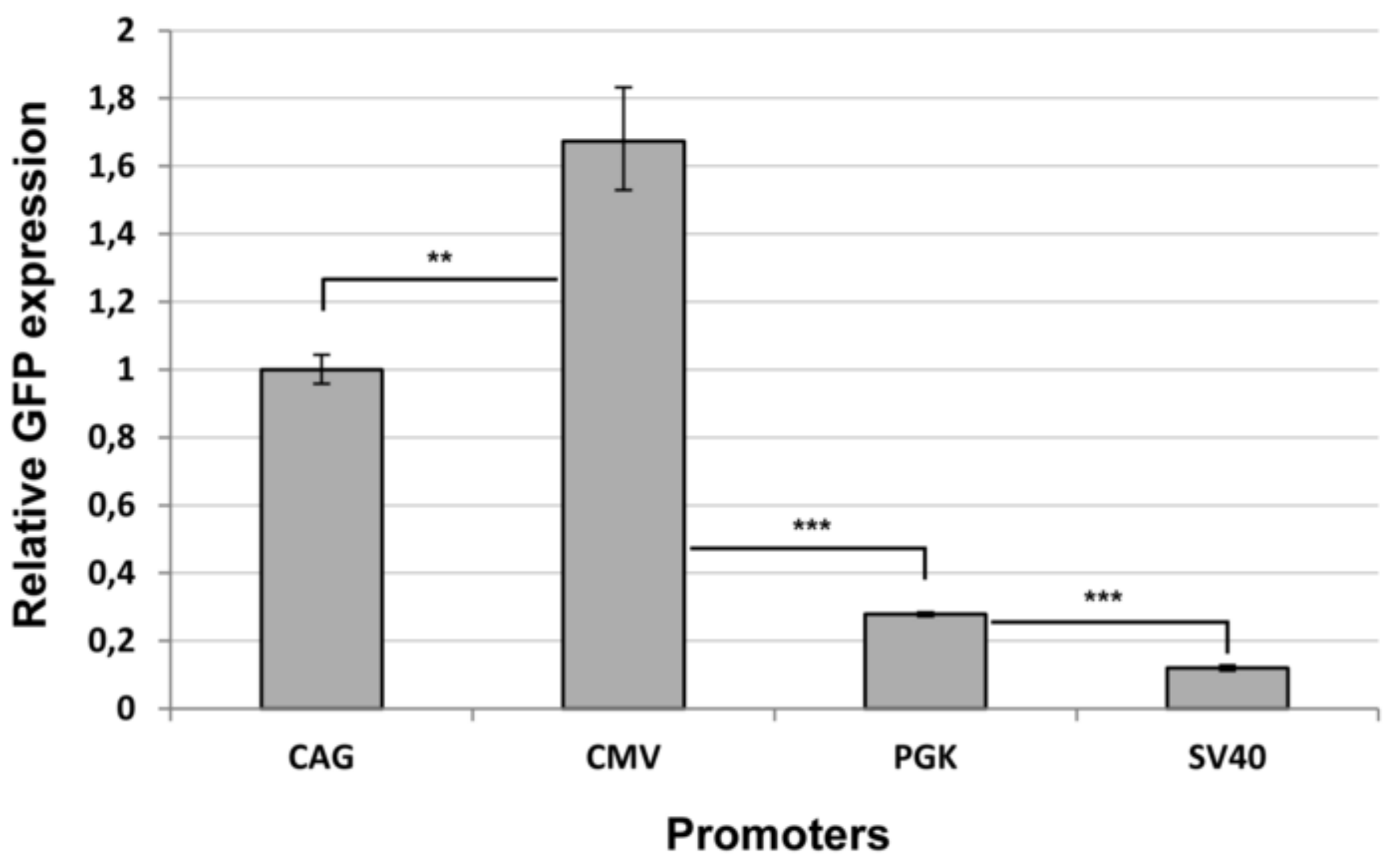

Figure 2 
Click here to download high resolution image

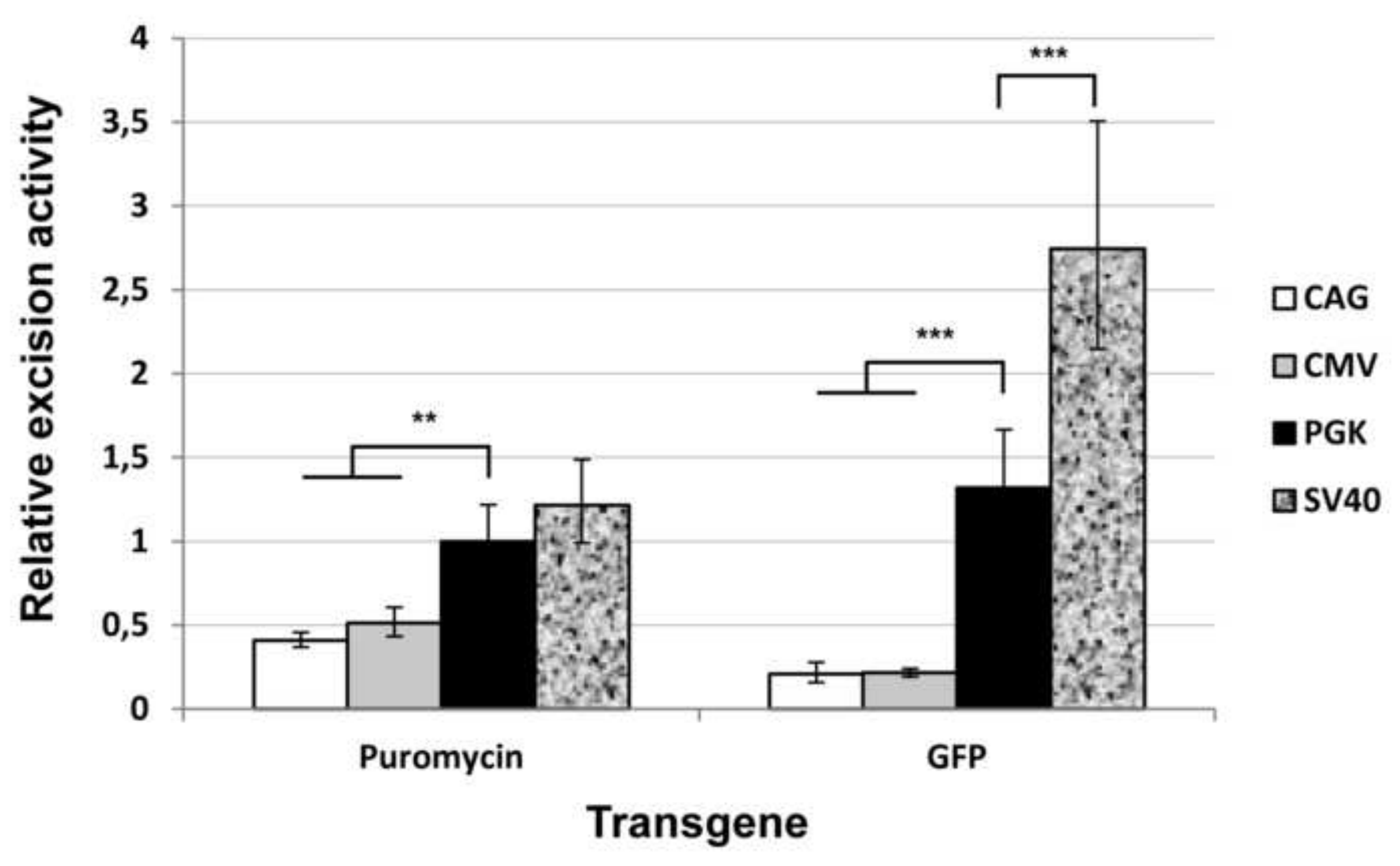

Figure 3 
Click here to download high resolution image

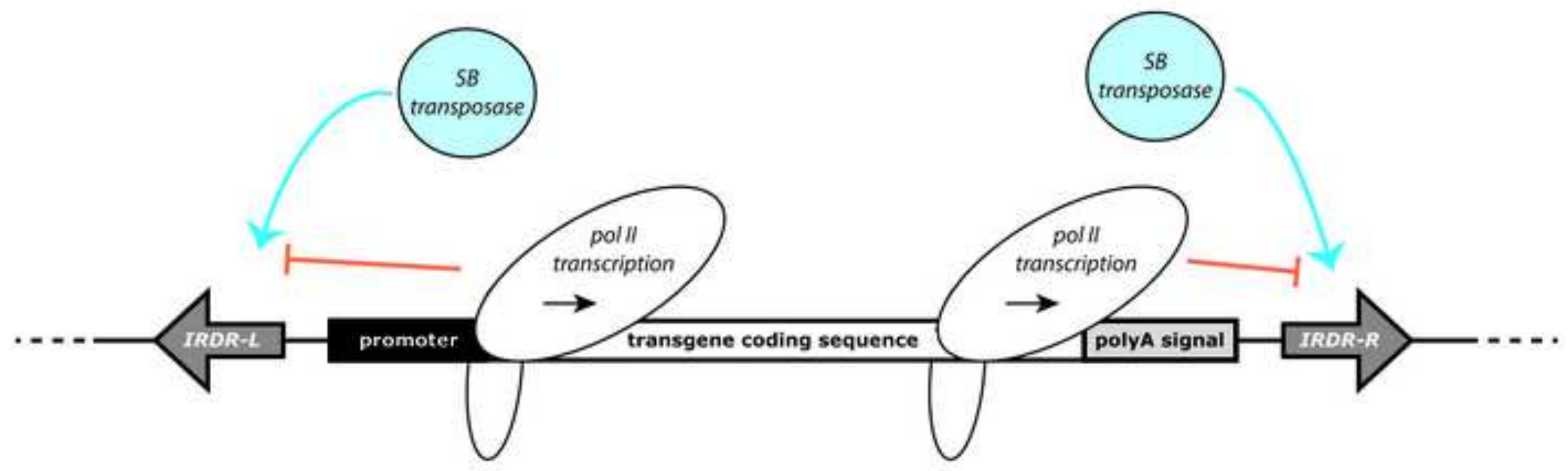

